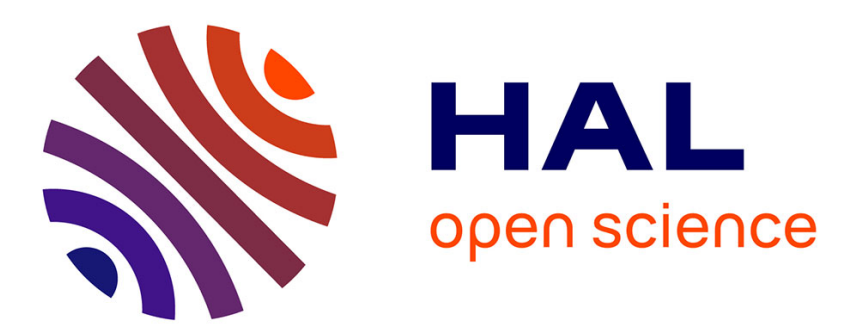

\title{
Coupling wavelets/vaguelets and smooth fictitious domain methods for elliptic problems: the univariate case
}

Ping Yin, Jacques Liandrat

\section{- To cite this version:}

Ping Yin, Jacques Liandrat. Coupling wavelets/vaguelets and smooth fictitious domain methods for elliptic problems: the univariate case. Computational \& Applied Mathematics, 2016, pp.DOI: 10.1007/s40314-014-0136-9. 10.1007/s40314-014-0136-9 . hal-01108936

\section{HAL Id: hal-01108936 \\ https://hal.science/hal-01108936}

Submitted on 1 Feb 2016

HAL is a multi-disciplinary open access archive for the deposit and dissemination of scientific research documents, whether they are published or not. The documents may come from teaching and research institutions in France or abroad, or from public or private research centers.
L'archive ouverte pluridisciplinaire HAL, est destinée au dépôt et à la diffusion de documents scientifiques de niveau recherche, publiés ou non, émanant des établissements d'enseignement et de recherche français ou étrangers, des laboratoires publics ou privés. 


\title{
Coupling wavelets/vaguelets and smooth fictitious domain methods for elliptic problems; the univariate case
}

\author{
Ping Yin ${ }^{1}$ \\ Science college, Jiangnan University, Wuxi 214122, China \\ Jacques Liandrat* \\ Centrale Marseille, I2M, UMR 7373, CNRS, Aix-Marseille Univ., Marseille, 13453, \\ France
}

\begin{abstract}
This work is devoted to the definition, the analysis and the implementation in the univariate case of a new numerical method for the approximation of partial differential equations solutions defined on complex domains. It couples a smooth fictitious domain method of Haslinger et al. [Projected Schur complement method for solving non-symmetric systems arising from a smooth fictitious domain approach, Numer. Linear Algebra 14(2007) 713-739] with multiscale approximations. After the definition of the method, error estimates are derived: they allow to control a global error (on the whole domain including the boundary of the initial complex domain) as well as an interior error (for any sub-domain strictly included in the control domain). $\mathrm{Nu}-$ merical implementation and tests on univariate elliptic problems are finally described.
\end{abstract}

Keywords: smooth fictitious domain method; multiscale approximation; error estimate; elliptic problem.

\footnotetext{
* Corresponding author

Email addresses: wanderapple@gmail.com (Ping Yin), jliandrat@centrale-marseille.fr (Jacques Liandrat)

${ }^{1}$ This work is supported by National Natural Science Foundation of China (No.11226307, 61100034), the Fundamental Research Funds for the Central universities (No.JUSRP 11213)
} 


\section{Introduction}

This paper is devoted to the numerical approximation of partial differential equations solutions posed on a non trivial domain. The general situation and notations, $\check{u}$ for the solution, $\omega$ for the domain and $\gamma$ for its boundary are shown in figures 1 and 2. It is well known that for variational formulations, the main difficulty lies in the construction of functional bases living on the domain $\omega$. For a time-dependent domain the difficulty reappears at each time step.

To circumvent this difficulty, fictitious domain methods ([1], [2] among many other references) have been introduced, leading to the definition of a new problem posed on a larger but simpler domain $\Omega \supset \omega$, called the fictitious domain, such that its solution $u$ restricted to $\omega$ matches with the solution $\breve{u}$ eventually when some so-called penalization parameter goes to zero. We summarize in the following the main difficulties encountered when one implements a variational approximation of fictitious domain methods:

1. Since the initial equation defined on $\omega$ is used to derive the new problem on the larger domain $\Omega$, its coefficients have to be extended. The choice of the extension is important since the regularity and eventually the support of the extended coefficients play a key role in the properties of the new equation and the numerical method used to approximate its solution.

2. In what will be called classical fictitious domain methods, the initial boundary condition is enforced on $\gamma$. This enforcing induces a lack of regularity of the solution in the vicinity of the domain where it is applied that weakens the convergence speed of the method [3].

3. Due to the presence of discontinuous coefficients or to the involvement of trace operators, ill conditioning is a standard property of numerical systems involved in fictitious domain methods.

4. So called inf/sup conditions [4] are usually associated to the weak formulations of fictitious domain methods that constrain the spaces of approximation involved in the implementation.

In this paper, we propose some answers to the above difficulties using two ingredients, the smooth fictitious domain method introduced in [3] and a multiscale approximation of wavelet type [5]. On one hand, we will show 
that the smooth fictitious domain method shifts the lack of regularity of the solution from the initial boundary to a new control boundary allowing an optimal convergence rate on the initial domain. On the other hand, we will show that the multiscale approach provides suitable answers to various open questions such as the extension procedure (1), the ill conditioning (3) and the local refinement demand associated to point (4).

The paper is organized as follows:

In section 2, we present preliminary results on wavelet approximation. The smooth fictitious domain method is presented in section 3 . Then, the details of our method coupling smooth fictitious domain method and wavelet approximation is described in section 4. In section 5, we describe the numerical implementation of our method and present detailed numerical results for univariate elliptic problems in section 6. Conclusion follows in section 7.

\section{Preliminary results on wavelet approximation}

We refer to [6] for a general introduction on wavelets and the notations used in the sequel. In the univariate situation, a sequence of nested approximation spaces $\left\{V_{j}\right\}_{j \in \mathbb{Z}}$ with additional conditions is used to build a multiresolution analysis of $L^{2}(\mathbb{R})$ or eventually of the Sobolev spaces $H^{s}(\mathbb{R}), s \in \mathbb{R}$. The function $\phi$ such that the set2 $\left\{\phi_{j, k}, j, k \in \mathbb{Z}\right\}$ is an Hilbertian basis of $V_{j}$ is called the scaling function. The orthogonal projection on $V_{j}$ is called $P_{j}$. The detail spaces between two successive approximation spaces are denoted by $W_{j}$, i.e., $V_{j+1}=V_{j} \bigoplus W_{j}$. All is designed such that $W_{j}=\operatorname{span}\left\{\psi_{j, k}, k \in \mathbb{Z}\right\}$ where the function $\psi$ is called the wavelet.

Two properties of a multi-resolution analysis will play a key role in the following; the regularity and the order that are related to the scaling function's smoothness and the polynomial reproduction quality of the spaces $V_{j}$. We will refer to them again when describing the smoothness of the functions in $V_{j}$ and the approximation quality of space $V_{j}$, that lead to so called Jackson and Bernstein inequalities.

We will use two constructions of wavelet bases of $L^{2}(\mathbb{R})$ leading to the Daubechies wavelets [7] and to the spline 3 wavelets [6].

\footnotetext{
${ }^{2}$ For any $f \in L^{2}(\mathbb{R})$ we note $f_{j k}(x)=2^{j / 2} f\left(2^{j} x-k\right)$ its dilated $\left(2^{j}\right)$ and translated $\left(k 2^{-j}\right)$ version

${ }^{3}$ For any segmentation $\left(x_{k}\right)_{0 \leq k \leq M}, x_{0}<x_{1}<\ldots<x_{M}$, splines of order $m \geq 2$ are $C^{m-1}$ functions $f$ such that, for each $k,\left.f\right|_{\left[x_{k}, x_{k+1}\right]}$ is a polynomial of degree $m-2$.
} 
Periodized multi-resolutions, multivariate multi-resolution analysis obtained by tensorial product and adaptation to compact support wavelet construction to the interval will also be used.

We are now ready to give the Jackson and Bernstein inequalities and their corollaries [8].

Theorem 1. (Jackson inequality)

Let $\left\{V_{j}\right\}_{j \in \mathbb{Z}}$ be the approximation spaces of a multi-resolution analysis of order $n ;$ Let $\Omega \subset \mathbb{R}^{d}$, if $f \in H^{s}(\Omega), 0<s \leq n$, then

$$
\left\|f-P_{j} f\right\|_{L^{2}(\Omega)} \leq C_{J, \phi^{\Omega}} 2^{-j s}\|f\|_{H^{s}(\Omega)},
$$

where the constant $C_{J, \phi^{\Omega}}$ depends only on the chosen multi-resolution analysis( the function $\phi^{\Omega}$ stands for the scaling function on the domain $\Omega$ ).

Corollary 2. Let $0<s<t \leq n$ and $n$ be the order of the multi-resolution analysis. If $f \in H^{t}(\Omega)$, then the following estimate holds

$$
\left\|f-P_{j} f\right\|_{H^{s}(\Omega)} \lesssim 2^{-j(t-s)}\|f\|_{H^{t}(\Omega)},
$$

here $A \lesssim B$ means that there exists a constant $c$ independent of $A$ and $B$ such that $A \leq c B$.

Theorem 3. (Bernstein inequality)

If $f \in V_{j}$, then one has

$$
\|f\|_{H^{s}(\Omega)} \leq C_{B, \phi^{\Omega}} 2^{s j}\|f\|_{L^{2}(\Omega)}, 0<s \leq m
$$

where $m$ is the multi-resolution analysis regularity. The constant $C_{B, \phi^{\Omega}}$ depends only on the chosen multi-resolution analysis.

Corollary 4. Let $0<s<t \leq m, m$ be the multi-resolution analysis' regularity and $n$ be the order of the multi-resolution analysis. If $s \leq n$ and $f \in V_{j}$, then

$$
\|f\|_{H^{t}(\Omega)} \lesssim 2^{(t-s) j}\|f\|_{H^{s}(\Omega)}
$$




\section{Smooth fictitious domain method; formulation and existence results}

\subsection{General formulation}

We consider first the different formulations of a general problem in two dimensions even if, from section 3.2 we focus on the univariate case. We consider a standard elliptic boundary-value problem defined on a bounded, open domain $\omega \subset \mathbb{R}^{2}$ equipped with Dirichlet boundary condition on its boundary $\gamma$ supposed to be Lipschitz continuous. It reads:

$$
\left\{\begin{aligned}
-\Delta \check{u}+\nu \check{u} & =\check{f} \text { in } \omega, \\
\check{u} & =g \text { on } \gamma,
\end{aligned}\right.
$$

where $\check{f} \in L^{2}(\omega)$ and $g \in H^{1 / 2}(\gamma)$ are given functions and $\nu$ is a positive constant. Its variational form is derived as

$$
\left\{\begin{array}{l}
\text { Find } \check{u} \in H^{1}(\omega), \text { such that } \check{u}=g \text { on } \gamma \text { and } \\
a_{\omega}(\check{u}, \check{v})=(\check{f}, \check{v})_{L^{2}(\omega)} \quad \forall \check{v} \in H_{0}^{1}(\omega),
\end{array}\right.
$$

where the bilinear operator $a_{\omega}(\cdot, \cdot)$ that maps $H^{1}(\omega) \times H^{1}(\omega)$ to $\mathbb{R}$ is defined as

$$
a_{\omega}(w, v)=\int_{\omega}(\nabla w \nabla v+\nu w v) d x .
$$

Fictitious domain methods substitute (21) by a new problem defined on a simpler domain $\Omega \supset \omega$ such that, by restriction to $\omega$ and eventually extension to the limit of some parameter (this is the case in penalization methods [2]), one can recover the solution of the initial problem. Here the solution of the fictitious domain method is constrained to have its trace on $\gamma$ satisfying in the weak sense the boundary condition of (2) using Lagrange multipliers. In classical fictitious domain methods for Dirichlet boundary conditions, the Lagrange multipliers belong then to $H^{-\frac{1}{2}}(\gamma)$ as introduced in [1]. Using $H_{p}^{1}(\Omega)$ a suitable subspace of $H^{1}(\Omega)$ we get:

$$
\left\{\begin{array}{l}
\text { Find } u \in H_{p}^{1}(\Omega), \lambda \in H^{-\frac{1}{2}}(\gamma), \quad \text { such that } \\
a_{\Omega}(u, v)+<T_{\gamma} v, \lambda>_{\gamma}=(f, v), \quad \forall v \in H_{p}^{1}(\Omega), \\
<T_{\gamma} u, \mu>_{\gamma}=<g, \mu>_{\gamma}, \quad \forall \mu \in H^{-\frac{1}{2}}(\gamma),
\end{array}\right.
$$

where $\langle\cdot, \cdot\rangle_{\gamma}$ denotes the duality pairing between $H^{\frac{1}{2}}(\gamma)$ and $H^{-\frac{1}{2}}(\gamma)$, $a_{\Omega}(\cdot, \cdot)$ has the same expression as (3) substituting $\omega$ by $\Omega$, the operator $T_{\gamma}$ 
represents the trace operator from $H^{1}(\Omega)$ to $H^{\frac{1}{2}}(\gamma)$ and $f$ is an extension of $\check{f}$ defined on $\Omega$. In our univariate applications, $H_{p}^{1}(\Omega)$ will be the subspace of periodic functions of $H^{1}[0,1]$.

The following remarks are required:

Remark 3.1. - This saddle point problem involves clearly an extension of the right-hand side term $\check{f}$ which is denoted by $f$. Without entering here into the details of the analysis we expect that the regularity of this extension constrains the regularity of the solution $u$ and therefore the convergence speed of any numerical method involved for the numerical approximation of (4). The effective construction of an extension raises various difficulties. Some of them will be addressed in section 5.2.3.

- It is well known ([9]) that, the solution of (4) suffers from a lack of regularity along the boundary $\gamma$ as soon as the Lagrange multiplier $\lambda$ is non zero.

To circumvent the second point, the so called smooth fictitious domain method has been introduced in [3]. In this method, the Lagrange multipliers used to enforce the boundary condition live on a control boundary $\Gamma \neq \gamma$ surrounding the domain $\omega$ and defining the control domain $\Xi$ (shown in figure 11). More precisely, the smooth fictitious domain formulation reads:

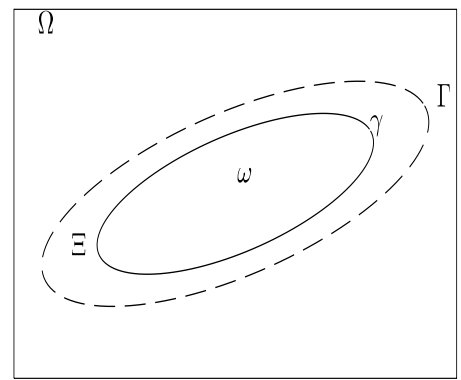

Figure 1: Geometry: $\omega$ is the original domain, $\gamma$ is its boundary. $\Gamma$ is the control boundary in the smooth fictitious domain method; $\Xi \supset \omega$ is bounded by $\Gamma$. The fictitious domain is called $\Omega$. 


$$
\left\{\begin{array}{lr}
\text { Find } u \in H_{p}^{1}(\Omega), \lambda \in H^{-\frac{1}{2}}(\Gamma), & \text { such that } \\
a_{\Omega}(u, v)+<T_{\Gamma} v, \lambda>_{\Gamma}=(f, v), & \forall v \in H_{p}^{1}(\Omega), \\
<T_{\gamma} u, \mu>_{\gamma}=<g, \mu>_{\gamma}, & \forall \mu \in H^{-\frac{1}{2}}(\gamma),
\end{array}\right.
$$

where $T_{\Gamma}$ stands for the trace operator from $H_{p}^{1}(\Omega)$ to $H^{\frac{1}{2}}(\Gamma)$ and $<\cdot, \cdot>_{\Gamma}$ is the pairing between $H^{\frac{1}{2}}(\Gamma)$ and its dual space $H^{-\frac{1}{2}}(\Gamma)$. If (5) has a solution $(u, \lambda)$, it is easy to check that $(u, \lambda)$ satisfies, in the weak sense, the following equations and boundary conditions:

$$
\left\{\begin{array}{l}
-\triangle u+\nu u=f \text { in } \Xi \cup(\Omega \backslash \Xi), \\
u=g \text { on } \gamma \\
\lambda=-\left[\frac{\partial u}{\partial n}\right]_{\Gamma} \text { on } \Gamma
\end{array}\right.
$$

where $n$ is the outward unit normal to $\Gamma$ and $[.]_{\Gamma}$ denotes the normal jump across $\Gamma$. The restriction of $u$ on $\omega$ therefore satisfies (1) in the weak sense.

Concerning the regularity of $u$, the last equation of (6) shows that $u$ is globally constrained in the space $H^{\frac{3}{2}-\epsilon}(\Omega)$ as soon as the Lagrange multipliers $\lambda$ is non zero. However, on $\Xi$, according to (6),$u \in H^{m+2}(\Xi)$ as soon as $f \in H^{m}(\Xi)$. One should notice that this situation depends directly on the smoothness of the extension of $f$ in $\Omega \backslash \bar{\omega}$. Moreover, equation (5) provides a smooth extension of the solution of (1) on $\Xi$. This solution is smoother than the restriction to $\Xi$ of the solution of the classical fictitious domain method (4) that indeed belongs to $H^{\frac{3}{2}-\epsilon}(\Xi)$ due to the discontinuity of $\frac{\partial u}{\partial n}$ on $\gamma$.

Therefore, one can expect that locally faster convergence of the numerical methods can be reached (see the next sections).

This advantage of the smooth fictitious domain method is however not given for free: indeed, it appears that there does not exist solution $(u, \lambda) \in$ $H^{1}(\Omega) \times H^{-\frac{1}{2}}(\Gamma)$ for arbitrary $(f, g) \in L^{2}(\Omega) \times H^{\frac{1}{2}}(\gamma)$. Indeed, if this was the case, classical interior estimates for elliptic equations show that $u \in H^{2}(O)$ for any open subset of $O \subset \Xi$ and ,among them, for any subset containing $\bar{\omega}$. Therefore, according to the trace theorem on the interior of $O$, the trace of $u$ on $\gamma$ belongs to $H^{\frac{3}{2}}(\gamma)$. This is not compatible with the initial hypothesis $g \in H^{\frac{1}{2}}(\gamma)$.

J.Haslinger and co-authors [3] indeed pointed out that the problem (5) is related to the controllability of the initial problem. A classical result of control theory asserts that the system controllability is only approximative, 
that means that the smooth fictitious domain method resulting problem has a solution only when $g$ belongs to a dense subset of $H^{\frac{1}{2}}(\gamma)$. As it is shown in the next section the situation is simpler in the univariate case.

\subsection{Specific analysis for the univariate case}

We analyze the univariate version of problem (15) in the framework of existence of solution for generalized saddle point problem [10]. Assuming that $\Omega=[0,1], \omega=] a, b[, \Xi=] a^{\prime}, b^{\prime}\left[\right.$ with $0<a^{\prime}<a<b<b^{\prime}<1$ as shown in figure 2, the formulation (5) is specified as follows:

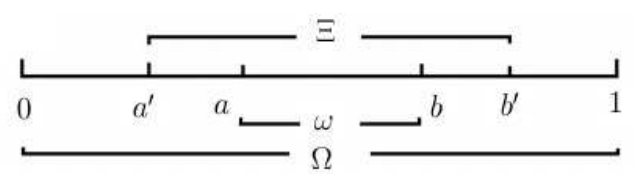

Figure 2: Geometry in 1D

$$
\left\{\begin{array}{l}
\text { Find } u \in H_{p}^{1}[0,1], \lambda=\left(\lambda_{1}, \lambda_{2}\right) \in \mathbb{R}^{2}, \\
a_{\Omega}(u, v)+\lambda_{1} v\left(a^{\prime}\right)+\lambda_{2} v\left(b^{\prime}\right)=(f, v), \quad \forall v \in H_{p}^{1}[0,1] \\
u(a)=g(a), \quad u(b)=g(b)
\end{array}\right.
$$

For convenience in the sequel, we note

$$
\begin{aligned}
& L_{1}^{\sigma}=\left\{u \in H_{p}^{1}(\Omega) \text { such that }\left.u\right|_{\partial \omega}=\sigma, i . e, u(a)=\sigma(a), u(b)=\sigma(b)\right\}, \\
& L_{2}^{\sigma}=\left\{u \in H_{p}^{1}(\Omega) \text { such that }\left.u\right|_{\partial \Xi}=\sigma, i . e, u\left(a^{\prime}\right)=\sigma\left(a^{\prime}\right), u\left(b^{\prime}\right)=\sigma\left(b^{\prime}\right)\right\} .
\end{aligned}
$$

By Proposition 7.1.4 [11], it is well-known that problem (7) can be written as :

$$
\text { Find } u \in L_{1}^{g} \text {, such that } a_{\Omega}(u, v)=(f, v), \forall v \in L_{2}^{0} \text {. }
$$

Existence and uniqueness of the solution of (9) depends on the existence of a positive constant $\delta$ such that

$$
\sup _{v \in L_{2}^{0}} \frac{a_{\Omega}(u, v)}{\|v\|_{H_{p}^{1}(\Omega)}} \geq \delta\|u\|_{H_{p}^{1}(\Omega)}, \forall u \in L_{1}^{0}
$$


Remark 3.2. In the classical fictitious domain method, the equation equivalent to (10) is obtained inserting $L_{1}^{0}=L_{2}^{0}$. Since $a_{\Omega}$ is coercive the condition is trivially satisfied.

Without loss of generality, we focus on a simplified version of (10) involving $H_{r}^{1}=\left\{u \in H^{1}[0,1] \mid u(1)=0\right\}, H_{l}^{1}=\left\{u \in H^{1}[0,1] \mid u(0)=0\right\}$ and $a_{\Omega}(u, v)=(u, v)_{H^{1}(\Omega)}$. We then have the following lemma:

Lemma 5. For any $u \in H_{r}^{1}$, there exists a positive constant $c$ such that

$$
\sup _{v \in H_{l}^{1}} \frac{(u, v)_{H^{1}(\Omega)}}{\|v\|_{H^{1}(\Omega)}} \geq c\|u\|_{H^{1}(\Omega)}, \forall u \in H_{r}^{1} .
$$

\section{Proof:}

By Lax-Milgram theorem, there exists a unique element $T u \in H_{l}^{1}$ such that

$$
(T u, v)_{H^{1}(\Omega)}=(u, v)_{H^{1}(\Omega)}, \forall v \in H_{l}^{1} .
$$

Moreover, $T u-u$ satisfies

$$
\left\{\begin{array}{r}
-(T u-u)^{\prime \prime}+(T u-u)=0 \\
(T u-u)(0)=-u(0), \\
(T u-u)^{\prime}(1)=0 .
\end{array}\right.
$$

A direct calculation gives $T u=u+A e^{x}+B e^{-x}$, with $A=-\frac{u(0)}{1+e^{2}}$ and $B=-\frac{u(0) e^{2}}{1+e^{2}}$, moreover,

$$
\|T u\|_{H^{1}}^{2}=\|u\|_{H^{1}}^{2}-\frac{e^{2}-1}{e^{2}+1}(u(0))^{2} .
$$

Since $\int_{0}^{1} u^{\prime}(x) d x=-u(0)$, we have $|u(0)| \leq\|u\|_{H^{1}}$. Using this inequality in (14),

$$
\|T u\|_{H^{1}(\Omega)} \geq c\|u\|_{H^{1}(\Omega)}
$$

with $c=\sqrt{\frac{2}{e^{2}+1}}$. Finally,

$$
\sup _{v \in H_{l}^{1}} \frac{(u, v)_{H^{1}(\Omega)}}{\|v\|_{H^{1}(\Omega)}} \geq \frac{(u, T u)_{H^{1}(\Omega)}}{\|T u\|_{H^{1}(\Omega)}} \geq c\|u\|_{H^{1}(\Omega)},
$$

that concludes the proof.

Following [10], lemma 5 leads to the following theorem:

Theorem 6. In the univariate situation, there exists a unique solution to (1) and the initial problem has exact controllability. 


\section{Wavelet approximation}

Following [12], we choose a Petrov-Galerkin and a wavelet-vaguelet method to discretize (5). We denote by $\left\{U_{j}^{\Omega}\right\}_{j \in \mathbb{Z}}$ a $m \geq 1$ regular and $n \geq 1$ order orthogonal multi-resolution of $H_{p}^{1}[0,1]$, the subset of $H^{1}[0,1]$ of periodic elements. We define $V_{j}^{\Omega}=(-\triangle+\nu I)^{-1} U_{j}^{\Omega}, j \in \mathbb{Z}$. The family $\left\{V_{j}^{\Omega}\right\}_{j \in \mathbb{Z}}$ is a bi-orthogonal multi-resolution spanned by vaguelets that inherits the important properties of classical multi-resolution by keeping the vanishing moments and decrease property of its natural bases, as seen in [13]. Similarly, $Q_{j^{\prime}}^{\Gamma}\left(\operatorname{resp} . Q_{j^{\prime \prime}}^{\gamma}\right)$ is a $m^{\prime} \geq 1$ (resp. $m^{\prime \prime} \geq 1$ ) regular and $n^{\prime} \geq 1$ order(resp. $n^{\prime \prime} \geq 1$ ) orthogonal multi-resolution of $H^{-1 / 2}(\Gamma)\left(\right.$ resp. $\left.H^{-1 / 2}(\gamma)\right)$.

The discretized problem then reads:

$$
\left\{\begin{array}{lr}
\text { Find } u_{j} \in U_{j}^{\Omega}, \lambda_{j^{\prime}} \in Q_{j^{\prime}}^{\Gamma}, \text { such that } & \\
a_{\Omega}\left(u_{j}, v_{j}\right)+<T_{\Gamma} v_{j}, \lambda_{j^{\prime}}>_{\Gamma}=\left(f, v_{j}\right) & \forall v_{j} \in V_{j}^{\Omega}, \\
<T_{\gamma} u_{j}, \mu_{j^{\prime \prime}}>_{\gamma}=<g, \mu_{j^{\prime \prime}}>_{\gamma} & \forall \mu_{j^{\prime \prime}} \in Q_{j^{\prime \prime}}^{\gamma} .
\end{array}\right.
$$

The existence of a unique solution $\left(u_{j}, \lambda_{j^{\prime}}\right)$ to (16) is here taken for granted. It will be proved in Section 5.3, Remark 5.1.

\subsection{Global error estimate}

According to [12], the approximation error $u-u_{j}$ satisfies the following global error estimate:

If $u \in H^{s}(\Omega), 0<s \leq n, \lambda \in H^{s^{\prime}}(\Gamma), 0<s^{\prime} \leq n^{\prime}$, then

$$
\left\|u-u_{j}\right\|_{H^{1}(\Omega)} \lesssim 2^{-j(s-1)}\|u\|_{H^{s}(\Omega)}+2^{-j^{\prime} s^{\prime}}\|\lambda\|_{H^{s^{\prime}}(\Gamma)}
$$

and

$$
\left\|u-u_{j}\right\|_{L^{2}(\Omega)} \lesssim 2^{-j s}\|u\|_{H^{s}(\Omega)}+2^{-j^{\prime} s^{\prime}}\|\lambda\|_{H^{s^{\prime}(\Gamma)}} .
$$

\subsection{Interior error estimate}

The above estimates involve the full space $\Omega$ and are therefore called global estimates. In our approach involving Lagrange multipliers on the control boundary $\Gamma$, as well as in the classical fictitious domain method where the Lagrange multipliers have support on $\gamma$, the regularity of the solution $u$ is limited to $H^{\frac{3}{2}-\epsilon}$, for all $\epsilon>0$ as soon as the Lagrange multipliers are non zero. However, on any open set $O$ with no intersection with the boundary where the normal derivative gets a jump, it is a classical result that $u \in H^{m+2}(O)$ 
if $f \in H^{m}(O)$. In the smooth fictitious domain method this is true for any open subset of $\Xi$. This local high regularity of the solution suggests that one can get for compact support approximation methods a better local rate of convergence of the approximation. Indeed, it has been shown by S.Bertoluzza [9] that a Galerkin method based on compactly supported wavelets satisfies the general assumptions, related to the discretization spaces, sufficient to ensure some so called interior error estimate as derived in [14].

The proof involves two key ingredients: the compact support property of the involved wavelet basis and the multi-resolution regularity and order under which the Jackson and Bernstein type inequalities and norm equivalences follow. In our case, due to the implementation of a Petrov Galerkin method and since the operator $-\Delta+\nu I$ is non-homogeneous, the basis of the discretization space $V_{h}^{\Omega}$ is not compactly supported and any interior error estimate can not be reached by a direct generalization of [9].

However, we will be able to derive an error estimate comparable to the one in [9] using two steps: first, modifying the right-hand side term of (1), we will show that the solution $\check{u}$ is also solution of another problem involving a homogeneous operator. A Petrov Galerkin method involving compactly supported bases and the generalization of the results of [9] can be derived, leading to lemma 7, second, the comparison of the approximated solutions for the two problems allows to derive a new result (theorem 10). This result involves the distance between the initial boundary and the control boundary, a parameter that indeed appears naturally in our numerical results (see section 6).

Step 1: New problem and corresponding interior estimate

Starting from the extension of (11) on $[0,1]$, we first consider the following problem:

$$
\left\{\begin{array}{l}
-\frac{\partial^{2}}{\partial x^{2}} \bar{u}=\bar{f}, \text { in }[0,1], \\
\bar{u}(a)=g(a), \quad \bar{u}(b)=g(b),
\end{array}\right.
$$

where the right-hand side term is defined through its Fourier transform 4 as $\hat{\hat{f}_{k}}=\frac{4 \pi^{2} \xi^{2} \hat{f}_{k}}{\nu+4 \pi^{2} k^{2}}$.

\footnotetext{
${ }^{4}$ for all $f \in L^{2}(] 0,1[)$ its Fourier transform is defined as for all $k \in \mathbb{Z}, \hat{f}_{k}=$ $\int_{0}^{1} f(x) e^{-i 2 \pi k x} d x$; for all $f \in L^{2}(\mathbb{R}), \hat{f}(\xi)=\int_{\mathbb{R}} f(x) e^{-i \xi x} d x$.
} 
Applying the smooth fictitious domain method to (19) with $\Xi=] a^{\prime}, b^{\prime}[\supset$ ]$a, b[$ leads to the following problem:

$$
\left\{\begin{array}{l}
\text { Find } \bar{u} \in H_{p}^{1}[0,1], \bar{\lambda}=\left(\bar{\lambda}_{1}, \bar{\lambda}_{2}\right) \in \mathbb{R}^{2}, \quad \text { such that } \\
a_{1}(\bar{u}, \bar{v})+\bar{\lambda}_{1} \bar{v}\left(a^{\prime}\right)+\bar{\lambda}_{2} \bar{v}\left(b^{\prime}\right)=(\bar{f}, \bar{v}), \quad \forall \bar{v} \in H_{p}^{1}[0,1], \\
\bar{u}(a)=g(a), \quad \bar{u}(b)=g(b),
\end{array}\right.
$$

where $a_{1}(\bar{u}, \bar{v})=\int_{0}^{1} \frac{\partial \bar{u}}{\partial x} \frac{\partial \bar{v}}{\partial x} d x$.

We introduce

$$
U_{j}^{[0,1]}=\operatorname{span}\left\{\phi_{j_{0}, k}, \psi_{l, k}, k \in \mathbb{Z}, j_{0} \leq l<j\right\},
$$

where we assume that all the functions $\phi_{j_{0}, k}, \psi_{l, k}$ satisfy a dilation-translationcompact-support condition that reads:

there exists a constant $\mathrm{N}$ such that for any $k \in \mathbb{Z}$

$$
\begin{gathered}
\operatorname{supp} \phi_{j_{0}, k} \subset\left[(k-N) 2^{-j_{0}},(k+N) 2^{-j_{0}}\right], \\
\operatorname{supp} \psi_{l, k} \subset\left[(k-N) 2^{-l},(k+N) 2^{-l}\right] .
\end{gathered}
$$

This condition is satisfied as soon as these functions are obtained by dilations and translations of compactly supported functions $\phi$ and $\psi$. Moreover we assume that the spaces $\left\{U_{j}^{[0,1]}\right\}_{j \in \mathbb{Z}}$ constitute a multi-resolution analysis $\mathrm{f}$ $L^{2}[0,1]$ of regularity $m$ and order $n$.

Following the implementation of the Petrov-Galerkin method [12] leads to consider the function $\varphi$ defined by $\hat{\varphi}(\xi)=\sin ^{2}(2 \pi \xi) \hat{\phi}(\xi)$ as well as the functions

$$
\begin{gathered}
\tilde{\theta}_{l, k}(x)=\left(-\frac{\partial^{2}}{\partial x^{2}}\right)^{-1} \psi_{l, k}, \\
\tilde{\vartheta}_{j_{0}, k}(x)=\left(-\frac{\partial^{2}}{\partial x^{2}}\right)^{-1} \varphi_{j_{0}, k},
\end{gathered}
$$

that generate the space

$$
\bar{V}_{j}^{[0,1]}=\operatorname{span}\left\{\tilde{\vartheta}_{j_{0}, k}, \tilde{\theta}_{l, k}, k \in \mathbb{Z}, j_{0} \leq l<j\right\} .
$$

Therefore the discretization form of (20) reads:

$$
\left\{\begin{array}{l}
\text { Find } \quad \bar{u}_{j} \in U_{j}^{[0,1]}, \bar{\lambda}_{j}=\left(\bar{\lambda}_{j, 1}, \bar{\lambda}_{j, 2}\right) \in \mathbb{R}^{2}, \text { such that } \\
a_{1}\left(\bar{u}_{j}, \bar{v}_{j}\right)+\bar{\lambda}_{j, 1} \bar{v}_{j}\left(a^{\prime}\right)+\bar{\lambda}_{j, 2} \bar{v}_{j}\left(b^{\prime}\right)=\left(\bar{f}, \bar{v}_{j}\right), \quad \forall \bar{v}_{j} \in \bar{V}_{j}^{[0,1]} \\
\bar{u}_{j}(a)=g(a), \quad \bar{u}_{j}(b)=g(b),
\end{array}\right.
$$


Note that, as mentioned in [15], the two spaces $U_{j}^{[0,1]}$ and $\bar{V}_{j}^{[0,1]}$ are both generated by compactly supported bases of wavelets or vaguelets.

We then have the following Lemma:

Lemma 7. Let $\omega_{0} \subset \omega_{1} \subset[0,1], \bar{u} \in H_{p}^{l}[0,1], \bar{u}_{h} \in U_{j}^{[0,1]}$ and $p$ be a nonnegative integer, arbitrary but fixed. The approximation space $\bar{V}_{j}^{[0,1]}$ is constructed as previously. Then there exists $\tilde{j}$, for all $j_{0}>\tilde{j}$, we have

$$
\left\|\bar{u}-\bar{u}_{h}\right\|_{s, \omega_{0}} \lesssim 2^{-j(l-s)}\|\bar{u}\|_{l, \omega_{1}}+\left\|\bar{u}-\bar{u}_{h}\right\|_{-p, \omega_{1}}, \quad 1 \leq l \leq n, s=0,1,
$$

where $n$ denotes the order of the multi-resolution analysis $U_{j}^{[0,1]}$.

\section{Proof:}

The proof mimics the ones of Theorem 5.1 [14] and Theorem 2.1 [9]. The essential ingredients are the compact support property of the bases of $U_{j}^{[0,1]}$ and $\bar{V}_{j}^{[0,1]}$ and the fact that the spaces $\bar{V}_{j}^{[0,1]}$ are generated by vaguelets and therefore inherits the essential properties of a classical multi-resolution analysis which ensure that the Jackson estimate, Bernstein estimate and norm equivalence hold.

Step 2: Comparison of the two approximate solutions

We recall that the weak formulation of problem (11) is

$$
\left\{\begin{array}{l}
\text { Find } u \in H_{p}^{1}[0,1], \lambda=\left(\lambda_{1}, \lambda_{2}\right) \in \mathbb{R}^{2}, \quad \text { such that } \\
a_{2}(u, v)+\lambda_{1} v\left(a^{\prime}\right)+\lambda_{2} v\left(b^{\prime}\right)=(f, v), \quad \forall v \in H_{p}^{1}[0,1] \\
u(a)=g(a), \quad u(b)=g(b),
\end{array}\right.
$$

where $a_{2}(u, v)=\int_{0}^{1}\left(\nu u v+\frac{\partial u}{\partial x} \frac{\partial v}{\partial x}\right) d x$. As above we introduce

$$
\begin{gathered}
\theta_{l, k}(x)=\left(-\frac{\partial^{2}}{\partial x^{2}}+\nu I\right)^{-1} \psi_{l, k}(x), \\
\vartheta_{j_{0}, k}(x)=\left(-\frac{\partial^{2}}{\partial x^{2}}+\nu I\right)^{-1} \varphi_{j_{0}, k}(x),
\end{gathered}
$$

and the approximation space

$$
V_{j}^{[0,1]}=\operatorname{span}\left\{\vartheta_{j_{0}, k}, 0 \leq k \leq 2^{j_{0}}-1, \theta_{l, k}, j_{0} \leq l \leq j-1,0 \leq k \leq 2^{l}-1\right\} .
$$


The discrete form of (22) is now

$$
\left\{\begin{array}{l}
\text { Find } u_{j} \in U_{j}^{[0,1]}, \lambda_{j}=\left(\lambda_{j, 1}, \lambda_{j, 2}\right) \in \mathbb{R}^{2}, \quad \text { such that } \\
a_{2}\left(u_{j}, v_{j}\right)+\lambda_{j, 1} v_{j}\left(a^{\prime}\right)+\lambda_{j, 2} v_{j}\left(b^{\prime}\right)=\left(f, v_{j}\right), \quad \forall v_{j} \in V_{j}^{[0,1]}, \\
u_{j}(a)=g(a), \quad u_{j}(b)=g(b) .
\end{array}\right.
$$

The comparison of $u_{j}$, the solution of (23) and $\bar{u}_{j}$, the solution of (21) will provide the expected result. First, we recall from [6] the following Lemma about the decay of the functions $\theta_{l, k}(x)$ and $\vartheta_{j_{0}, k}(x)$.

Lemma 8. ([6] ] With $\theta_{l, k}$ and $\vartheta_{j_{0}, k}$ previously defined, the following inequalities hold for any $q \geq n$ where $n$ is the order of the multi-resolution:

$$
\begin{aligned}
\left|2^{2 l} \theta_{l, k}(x)\right| & \lesssim \frac{2^{l / 2}}{\left(1+2^{l}|x-k|\right)^{q}}, \\
\left|2^{2 j_{0}} \vartheta_{j_{0}, k}(x)\right| & \lesssim \frac{2^{j_{0} / 2}}{\left(1+2^{j_{0}}|x-k|\right)^{q}} .
\end{aligned}
$$

We have also the following inequality:

Lemma 9. Let $u, \bar{u}, \varphi$ be defined as previously, then

$$
\sum_{0 \leq k \leq 2^{j_{0}}-1}\left|\left(u-\bar{u}, \phi_{j_{0}, k}\right)\right|^{2} \lesssim \sum_{0 \leq k \leq 2^{j_{0}}-1}\left|\left(u-\bar{u}, \varphi_{j_{0}, k}\right)\right|^{2} .
$$

\section{Proof:}

The definition of $\varphi_{j_{0}, k}$ implies that we have

$$
\begin{aligned}
\sum_{k}\left(u-\bar{u}, \varphi_{j_{0}, k}\right) \phi_{j_{0}, k} & =-\frac{1}{4} \sum_{k}\left(u-\bar{u}, \phi_{j_{0}, k+2}-2 \phi_{j_{0}, k}+\phi_{j_{0}, k-2}\right) \phi_{j_{0}, k} \\
& =-\frac{1}{4} \sum_{k}\left(u-\bar{u}, \phi_{j_{0}, k}\right)\left(\phi_{j_{0}, k+2}-2 \phi_{j_{0}, k}+\phi_{j_{0}, k-2}\right) \\
& =\sum_{k}\left(u-\bar{u}, \phi_{j_{0}, k}\right) \varphi_{j_{0}, k} \\
& =\frac{d^{2}}{d x^{2}} \sum_{k}\left(u-\bar{u}, \phi_{j_{0}, k}\right) \digamma_{j_{0}, k}
\end{aligned}
$$


where $\frac{d^{2}}{d x^{2}} \digamma_{j_{0}, k}=\varphi_{j_{0}, k}$ and $\hat{\digamma}_{j_{0}, k}(\xi)=-\frac{\hat{\phi}_{j_{0}, k}(\xi) \sin ^{2}\left(2 \pi \xi / 2^{j_{0}}\right)}{(2 \pi \xi)^{2}}$. Let us note $f_{0}=$ $\sum_{k \in \mathbb{Z}}\left(u-\bar{u}, \phi_{j_{0}, k}\right) \digamma_{j_{0}, k}$ and $f_{1}=\frac{d}{d x} f_{0}$. Then, $\frac{d}{d x} f_{1}=\sum_{k}\left(u-\bar{u}, \varphi_{j_{0}, k}\right) \phi_{j_{0}, k}$. Applying Poincaré inequality leads to

$\sum_{k}\left|\left(u-\bar{u}, \phi_{j_{0}, k}\right)\right|^{2} \lesssim\left\|f_{0}\right\|_{L^{2}[0,1]}^{2} \lesssim\left\|f_{1}\right\|_{L^{2}[0,1]}^{2} \lesssim\left\|\frac{d f_{1}}{d x}\right\|_{L^{2}[0,1]}^{2} \lesssim \sum_{k}\left|\left(u-\bar{u}, \varphi_{j_{0}, k}\right)\right|^{2}$

using the fact that $\left\{\digamma_{j_{0}, k}\right\}_{k}$ is a Riesz basis (theorem 2.2 [13]) and that $\left\{\phi_{j_{0}, k}\right\}_{k}$ is an orthonormal basis, that concludes the proof.

We are now ready to give the main theorem of this section concerning the interior error estimate.

Theorem 10. Let us consider $U_{j}^{[0,1]}, V_{j}^{[0,1]}$ and $\bar{V}_{j}^{[0,1]}$ constructed as previously and $\Xi=] a^{\prime}, b^{\prime}\left[\subset[0,1], \omega_{0}=\right] a_{0}, b_{0}\left[\subset \Xi, \omega_{0} \subset \omega_{1} \subset[0,1]\right.$. Let us assume that the multi-resolution $\left\{U_{j}^{[0,1]}\right\}_{j \in \mathbb{Z}}$ is of order $n$ and that $p$ is a nonnegative integer, arbitrary but fixed. If $\bar{u} \in H_{p}^{l}[0,1]$ and $u \in H_{p}^{1}[0,1]$, then there exists $\tilde{j}$ such that for all $j_{0}>\tilde{j}$, for all $q \geq n$

$$
\left\|u-u_{h}\right\|_{1, \omega_{0}} \lesssim 2^{-j(l-1)}\|\bar{u}\|_{l, \omega_{1}}+\left\|\bar{u}-\bar{u}_{h}\right\|_{-p, \omega_{1}}+2^{-q j_{0}} d^{-q},
$$

where $d=\min \left\{\left|a^{\prime}-a_{0}\right|,\left|b^{\prime}-b_{0}\right|\right\}, l \leq n$.

\section{Proof:}

We use the triangle inequality to get

$$
\left\|u-u_{h}\right\|_{1, \omega_{0}} \leq\|u-\bar{u}\|_{1, \omega_{0}}+\left\|\bar{u}-\bar{u}_{h}\right\|_{1, \omega_{0}}+\left\|\bar{u}_{h}-u_{h}\right\|_{1, \omega_{0}} .
$$

Then by the norm equivalence for wavelet decomposition [8], we have the upper bound of $\|u-\bar{u}\|_{1, \omega_{0}}$,

$$
\begin{aligned}
\|u-\bar{u}\|_{1, \omega_{0}} & \lesssim\left(\sum_{k}\left|\left(u-\bar{u}, \phi_{j_{0}, k}\right)\right|^{2}+\sum_{l \geq j_{0}} \sum_{k} 2^{2 l}\left|\left(u-\bar{u}, \psi_{l, k}\right)\right|^{2}\right)^{1 / 2} \\
& \lesssim\left(\sum_{k}\left|-\lambda_{1} \vartheta_{j_{0}, k}\left(a^{\prime}\right)-\lambda_{2} \vartheta_{j_{0}, k}\left(b^{\prime}\right)+\bar{\lambda}_{1} \tilde{\vartheta}_{j_{0}, k}\left(a^{\prime}\right)+\bar{\lambda}_{2} \tilde{\vartheta}_{j_{0}, k}\left(b^{\prime}\right)\right|^{2}\right. \\
& \left.+\sum_{l \geq j_{0}} \sum_{k} 2^{2 l}\left|-\lambda_{1} \theta_{l, k}\left(a^{\prime}\right)-\lambda_{2} \theta_{l, k}\left(b^{\prime}\right)+\bar{\lambda}_{1} \tilde{\theta}_{l, k}\left(a^{\prime}\right)+\bar{\lambda}_{2} \tilde{\theta}_{l, k}\left(b^{\prime}\right)\right|^{2}\right)^{1 / 2},
\end{aligned}
$$

where the second inequality is deduced from (22), (20) and Lemma 9. Note that since $\phi_{j_{0}, k}$ and $\psi_{l, k}$ are compactly supported, the number of terms appearing in the sum is finite. When the coarsest approximation level $j_{0}$ is 
large enough, $\tilde{\vartheta}_{j_{0}, k}\left(a^{\prime}\right), \tilde{\vartheta}_{j_{0}, k}\left(b^{\prime}\right), \tilde{\theta}_{l, k}\left(a^{\prime}\right)$ and $\tilde{\theta}_{l, k}\left(b^{\prime}\right)$ are equal to 0 as $d>0$. For the point values of functions $\vartheta_{j_{0}, k}$ and $\theta_{l, k}$ at $a^{\prime}$ and $b^{\prime}$, we use lemma 8 to get

$$
\begin{aligned}
\|u-\bar{u}\|_{1, \omega_{0}} & \lesssim\left(\sum_{k}\left|\frac{2^{j_{0} / 2}}{2^{4 j_{0}} d^{2}}\right|^{2}+\sum_{l \geq j_{0}} \sum_{k} 2^{2 l}\left|\frac{2^{l / 2}}{2^{(n+2) l} d^{n}}\right|^{2}\right)^{1 / 2} \\
& \lesssim 2^{-q j_{0}} d^{-q} .
\end{aligned}
$$

We use the same method to get the upper bound of the term $\left\|\bar{u}_{h}-u_{h}\right\|_{1, \omega_{0}}$.

Combined together with lemma 7, we have

$$
\left\|u-u_{h}\right\|_{1, \omega_{0}} \lesssim 2^{-j(l-1)}\|\bar{u}\|_{l, \omega_{1}}+\left\|\bar{u}-\bar{u}_{h}\right\|_{-p, \omega_{1}}+2^{-q j_{0}} d^{-q},
$$

that concludes the proof.

Following the same track we get also

$$
\left\|u-u_{h}\right\|_{0, \omega_{0}} \lesssim 2^{-j l}\|\bar{u}\|_{l, \omega_{1}}+\left\|\bar{u}-\bar{u}_{h}\right\|_{-p, \omega_{1}}+2^{-j_{0}(1+q)} d^{-q} .
$$

Remark 4.1. - A consequence of (24) is that the distance between the control boundary and original boundary influences the error estimate. However, when the parameter $p$ and $q$ are chosen large enough, the last two terms on the right-hand side of (24) become negligible. This leads to an optimal convergence rate.

- For the bivariate case, the track of the above proof can not be followed. Indeed point values are replaced by trace and scalar product on the control boundary. The generalization of theorem 10 to bivariate situations is still an open question.

\section{Numerical implementation}

\subsection{Approximation spaces}

In this section, we describe the approximation spaces used in our univariate simulations with $\Omega=[0,1], \omega=] a, b[$ and $\Xi=] a^{\prime}, b^{\prime}[$. 


\subsubsection{Multi-resolution of $H_{p}^{1}[0,1]$}

Spline multi-resolution has been used for the approximation of $H_{p}^{1}[0,1]$. We refer to [16] for the construction of orthonormal splines wavelets of order $m$. Explicit expressions for $\hat{\phi}(\omega), \hat{\psi}(\omega)$ are provided including there expansion in terms of translated versions of the basic spline function $B_{m}(x)$.

\subsection{Approximation of operators}

\subsubsection{Elliptic operator}

In addition to the initial basis of $H_{p}^{1}[0,1]$, the Petrov-Galerkin method involves the bi-orthogonal bases constructed applying the operator $\left(\nu I-\frac{\partial^{2}}{\partial x^{2}}\right)$ as explained in the previous section. These bases are explicitly constructed using the Fourier transform.

\subsubsection{Trace operator}

The value of a basic spline function $B_{m}(x)$ on any point of its compact support can be reached explicitly thanks to the classical relation recalled in footnote 5. Since, moreover spline scaling functions $\phi_{k}^{j}$ and wavelets $\psi_{k}^{j}$ are explicitly known in terms of the basic spline function, the computation of their value at any position and therefore of their trace is a simple and fast routine.

It has been shown in [17] that, up to any precision, there exists an index $r$ such that the space $V^{j+r}$ contains the bi-orthogonal functions $\theta_{k}^{j}$. Therefore, their trace can also be evaluated fast.

\subsubsection{Extension operator}

The numerical construction of an efficient extension operator from a set of functions defined on $\omega$ to a suitable set of functions defined on $\Omega \supset \omega$ is constrained by different requirements. More precisely, if we denote this extension operator $E_{\omega}^{\Omega}: \check{f} \in F(\omega) \mapsto f=E_{\omega}^{\Omega}(\check{f}) \in F(\Omega)$ with $f \mid \omega=\check{f}$, these constraints are:

1. The smoothness of $f$ must be of same order as the smoothness of $f$,

2. The support of $f$ must be as small as possible,

5

$$
\left\{\begin{array}{l}
B_{1}(x)=\chi_{[0,1]}, \\
B_{m}(x)=\frac{x}{m-1} B_{m-1}(x)+\frac{m-x}{m-1} B_{m-1}(x-1) .
\end{array}\right.
$$


3. The decomposition of $f$ on a suitable basis of $F(\Omega)$ must be obtained fast from the decomposition of $\check{f}$ on a suitable basis of $F(\Omega)$.

The importance of the smoothness requirement has been emphasized earlier. Constraints 2. and 3. are directly linked to the efficiency of the numerical implementation. An extension operator satisfying 1. and eventually 2. in specific situations, using Fourier expansion has been proposed in [18]. In [19], a new extension operator based on compactly supported basis of a multi-resolution analysis on the interval [20] has been derived. We provide the sketch of this extension in the sequel of the subsection for the univariate situation and $\omega=] a, b[\subset \Omega=[0,1]$.

Up to some rescaling and interpolation, the extension problem in our approximation framework consists in the following question: Knowing the expansion of a function $\check{f}$ in a finite number of elements of a compactly supported wavelet basis of $L^{2}[0,1]$, is it possible to construct a compactly supported function $f_{e}$ and, more precisely its expansion in a finite number of elements of a compactly supported Daubechies wavelet basis of $L^{2}(\mathbb{R})$ such that the restriction of $f_{e}$ on $[0,1]$ coincides with $f$.

Coming back to the construction of compactly supported wavelet bases on the interval defined in [21], it turns out that for scale parameter $j \geq 0$ in the case of Daubechies wavelet basis, the scaling functions generating the approximation space $V_{j}^{[0,1]}$ can be written explicitly as:

$$
\check{\phi}_{j, k}^{[0,1]}=\left.\sum_{m=-N}^{N+2^{j}} \eta_{m}^{j, k} \phi_{j, m}\right|_{[0,1]},
$$

where the coefficients $\eta_{m}^{j, k}$ are given in the construction. Clearly, removing the restriction to $[0,1]$ in the right-hand side term of (25) provides a compactly supported function of the space $V_{j}^{\mathbb{R}}$ coinciding with $\check{\phi}_{j, k}^{[0,1]}$ on $[0,1]$. In the sequel, we illustrate a comparison between this extension procedure, called MRA method, and another one, called spectral method, described in [18] and using Fourier approximation. In figure 3, the two extensions are constructed starting from the samplings at rate $h=2^{-6}$ of the restriction of the function $f(x)=\sin (2 \pi x)$ on $] a, b[=] 0,0.5[$. The main difference between these two methods lies in the unknown parameters computed: the unknown grid point values in the spectral method and the scaling coefficient in MRA method. We denote $f^{s}$ resp. $f^{M}$ the extension obtained by spectral and MRA method respectively. The function $f^{s}$ is of period 1 on $\mathbb{R}$. Due to the com- 
pact support of Daubechies wavelet, $f^{M}$ is moreover compactly supported. More precisely, in our case with $N=10, \operatorname{supp} f^{M} \subset[-0.3125,0.8125]$.

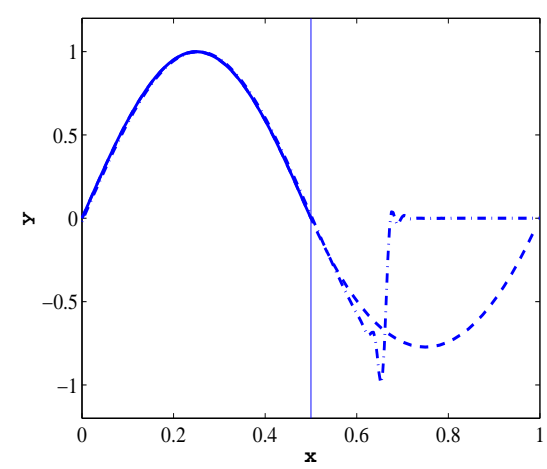

Figure 3: Function $\check{f}$ on initial domain $\omega$, denoted by solid line, and extended functions using spectral and MRA methods. The sampling points belong to the set $I=$ $\left\{\left(x_{i}, f\left(x_{i}\right)\right) \mid x_{i} \in[0,0.5], x_{i}=i h, h=1 / 2^{6}\right\}$ (on the left of the vertical line). The dashed line represents the extended function $f^{s}$ obtained using the spectral method with $p=1$, corresponding to a minimization procedure in $H^{2}[0,1]$. The dash-dotted line is the extended function $f^{M}$ using the MRA method with $N=10 ; f^{M} \in C^{2}[0,1]$.

\subsection{Numerical system and resolution}

Let us define $u_{j}=\sum_{k=0}^{2^{j}-1}\left(U_{j}\right)_{k} \phi_{k}^{j}, \Lambda_{j^{\prime}}=\left(\lambda_{1}, \lambda_{2}\right)$. The matrix form of (16) reads:

$$
\left\{\begin{array}{l}
\text { Find } U_{j} \in \mathbb{R}^{2^{j}}, \Lambda_{j^{\prime}} \in \mathbb{R}^{2}, \text { such that } \\
\left(\begin{array}{cc}
I & C \\
D & 0
\end{array}\right)\left(\begin{array}{c}
U_{j} \\
\Lambda_{j^{\prime}}
\end{array}\right)=\left(\begin{array}{c}
F_{j} \\
G_{j}
\end{array}\right)
\end{array}\right.
$$

with

$$
\begin{gathered}
\left(F_{j}\right)_{k}=\int_{0}^{1} f(x) \theta_{k}^{j}(x) d x \\
C=\left(\begin{array}{ccc}
\theta_{0}^{j}\left(a^{\prime}\right) & \theta_{0}^{j}\left(b^{\prime}\right) \\
\theta_{1}^{j}\left(a^{\prime}\right) & \theta_{1}^{j}\left(b^{\prime}\right) \\
\vdots & \vdots \\
\theta_{2^{j}-1}^{j}\left(a^{\prime}\right) & \theta_{2^{j}-1}^{j}\left(b^{\prime}\right)
\end{array}\right) \\
D=\left(\begin{array}{cccc}
\phi_{0}^{j}(a) & \phi_{1}^{j}(a) & \cdots & \phi_{2^{j}-1}^{j}(a) \\
\phi_{0}^{j}(b) & \phi_{1}^{j}(b) & \cdots & \phi_{2^{j}-1}^{j}(b)
\end{array}\right),
\end{gathered}
$$


and

$$
G_{j}=\left(\begin{array}{l}
u(a) \\
u(b)
\end{array}\right)
$$

Resolving this system (26) using Uzawa algorithm reads,

$$
\left\{\begin{array}{l}
\text { Find } U_{j} \in \mathbb{R}^{2^{j}}, \Lambda_{j^{\prime}} \in \mathbb{R}^{2}, \text { such that } \\
(D C)^{t} D C \Lambda_{j^{\prime}}=(D C)^{t} D F_{j}-(D C)^{t} G_{j}, \\
U_{j}=F_{j}-C \Lambda_{j^{\prime}} .
\end{array}\right.
$$

The next theorem provides bounds for the condition number of $(D C)^{t} D C$ proving that this system is well-conditioned.

Theorem 11. The matrix $(D C)^{t}(D C)$ satisfies the following estimate: $\exists 0<$ $K_{1}, K_{2}<+\infty$ such that for all $w=\left(w_{1}, w_{2}\right) \in \mathbb{R}^{2}$

$$
K_{1}(w, w)_{l^{2}} \leq\left((D C)^{t}(D C) w, w\right) \leq K_{2}(w, w)_{l^{2}}
$$

and therefore, for all $j$, cond $d_{2}\left((D C)^{t}(D C)\right) \leq \frac{K_{2}}{K_{1}}$.

\section{Proof:}

It mimics the proof of theorem 3.1 in [12] with an embedding theorem in $1 \mathrm{D}$ [11]. See [19] for details.

Remark 5.1. A direct consequence of Theorem 11 is that $(D C)^{t}(D C)$ is positive definite. Therefore system (26) is invertible and the problem (16) has a unique solution.

\section{Numerical results}

6.1. Comparison between smooth and classical multi-resolution-fictitious domain methods

We consider problem (11) with the following choices: $\left.\nu=\frac{1}{4 \pi^{2}}, \omega=\right] 0.2,0.5[$, $\check{u}=4 x^{2} \sin (2 \pi x)$ and therefore $\check{f}=(-\triangle+\nu I) \check{u}$ and $\check{g}=\left.\check{u}\right|_{\gamma}$. The fictitious domain is $\Omega=] 0,1[$ and the control domain is $\Xi=] 0.1,0.7[$. Splines of order 4 and scale $j=8$ are used. Figure 4 shows the numerical results obtained using the smooth (SFDM) and classical (CFDM) fictitious domain methods. Figure 5 shows the error versus $j$ in semi-logarithmic scale for different source 
term extensions $f$. For the classical methods the rate is limited by $-\frac{3}{2}$. For the smooth method a high convergence rate is reached, exceeding the result proven in our interior error estimate; it corresponds to a super convergence phenomena for multi-resolution methods that has already been observed, for instance in [17]. Note that, as expected, the smoothness of the extension plays an important role in the error decay.

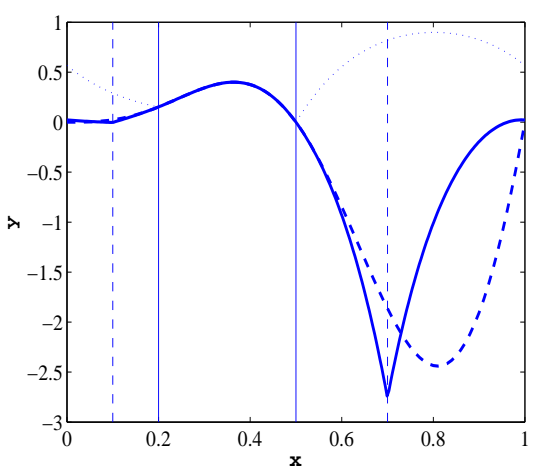

Figure 4: Numerical solution reached using classical (CFDM), denoted by dotted line, and smooth (SFDM) multi-resolution fictitious domain methods, denoted by solid line, using a constant extension compared to exact solution(denoted by dashed line). The solid vertical bounds the original domain $\omega$ while the dashed one bounds the control domain $\Xi$.

\subsubsection{Condition number}

The condition numbers of the iterative matrix $(D C)^{t}(D C)$ for different values of the scale $j$ is given in Table 1. As expected, they are numerically independent of the scale.

Table 1: condition number of matrix $(D C)^{t} D C$

\begin{tabular}{ll}
\hline $\mathrm{j}$ & $\operatorname{cond}_{2}(D C)^{t} D C$ \\
\hline 5 & 4.8525 \\
6 & 4.8494 \\
7 & 4.8496 \\
8 & 4.8496 \\
\hline
\end{tabular}




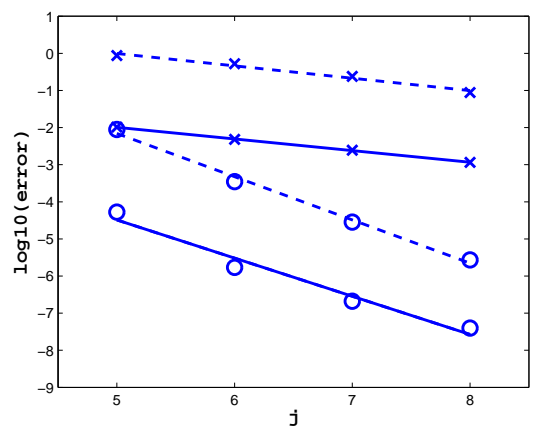

(a) constant extension

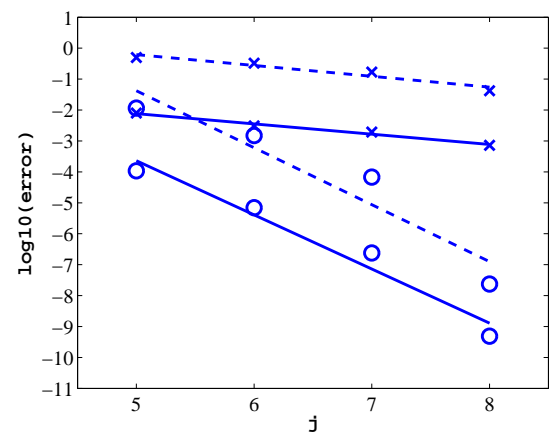

(b) $C^{\infty}$ extension

Figure 5: Error $\left\|u-u_{j}\right\|$ in norm $L^{2}(\omega)$ and $H^{1}(\omega)$ versus $j$ in semi-logarithmic scale. The circles stand for SFDM while cross stand for CFDM. The solid $\left(L^{2}(\omega)\right)$ and dashed $\left(H^{1}(\omega)\right)$ lines are the least square fits. On the left figure, the slopes from upper to bottom are $-1.1045,-1.0431,-3.8677,-3.1136$ while they are $-1.1677,-1.1062,-6.1177,-5.8137$ on the right figure.

\subsubsection{Influence of the distance between $\omega$ and $\Xi$ on the error rate}

To evaluate the effect of the distance $d$ between the boundary of $\omega$ and these of $\Xi$ on the error rate, we consider Problem (11) with $\nu=\frac{1}{64 \pi^{2}}$ and an exact solution $\check{u}=x^{4}-2 x^{3}+12 x^{2}-x+1$ on $\left.\omega=\right] 0.4,0.5[, \check{f}=(-\triangle+\nu I) \check{u}$, $\check{g}=\left.\check{u}\right|_{\gamma}$. Three different control domain are used, $\left.\Xi=\right] 0.3,0.6[, \Xi=] 0.2,0.7[$ and $\Xi=] 0.1,0.8[$ corresponding to $d=0.1,0.2$ and $d=0.3$. Figure 6 shows the error in $L^{2}(\omega)$ versus $j$ in semi-logarithmic scale for different $\Xi$ when $f$ is a constant or $C^{\infty}$ extension. As expected, the smoother is $f$, the faster is the decay of the error.

\section{Conclusions}

In this paper, we proposed and analyzed a new method which combines multi-resolution approximation and smooth fictitious domain method for elliptic problems. A full analysis has been performed in the univariate situation: proof of the existence and uniqueness of the solution and its approximation, definition and estimates of global and interior errors. A complete numerical method involving extension operator approximation has been provided. Compared to a classical fictitious domain method, a higher convergence rate is proved and various numerical advantages are exhibited on different tests. 


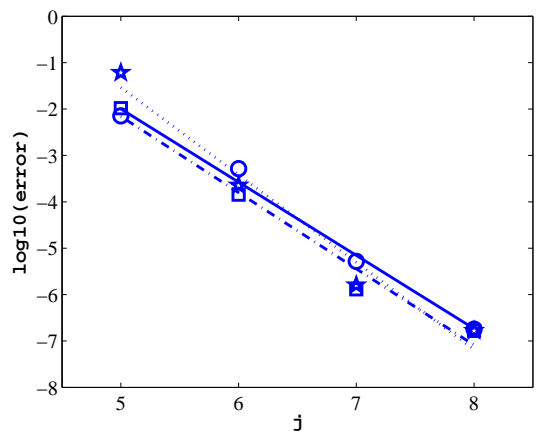

(a) constant extension

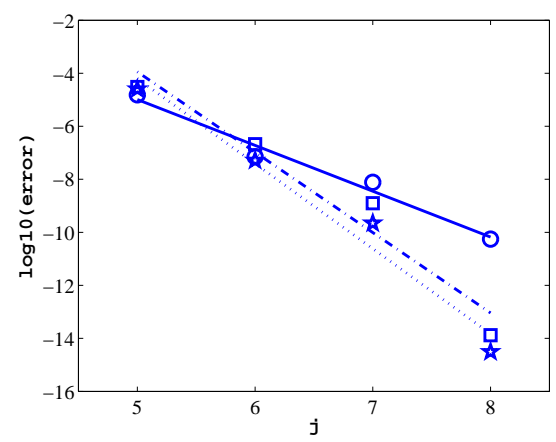

(b) $C^{\infty}$ extension

Figure 6: The error in norm $L^{2}(\omega)$ versus scale $\mathrm{j}$ with different $\Xi$ in semi-logarithmic scale. We use circle, square, pentagram to denote the error using $\Xi=] 0.3,0.6[, \Xi=$ ]0.2, $0.7[, \Xi=] 0.1,0.8[$ respectively. The solid, dash-dotted, dotted lines stands for their corresponding least square fits. On the left figure, the solid, dash-dotted, dotted line are of slope $-5.2453,-5.4576,-6.2575$. On the right figure, the solid, dash-dotted, dotted lines are of slope $-5.7449,-10.0684,-10.6624$.

In multidmension, the smooth fictitious domain method is well posed only for specific boundary conditions belonging to a dense subset of $H^{\frac{1}{2}}(\gamma)$ and the analysis of section 3 can not be generalized. However, our experience is that numerical approximation can be generalized in multidimension and our conjecture is that the convergence follows our results proved in the univariate case, including interior estimates; various numerical results can be found in ([19]).

Acknowledgments: Thanks are due to A. Benhabdalla and F. Boyer from I2M-UMR 7373 (France) for fruitful discussions on the control interpretation of the smooth fictitious domain method.

\section{References}

[1] J. Haslinger, J. Maitre, L. Tomas, Fictitious domain methods with distributed Lagrange multipliers. part i: application to the solution of elliptic state problems, Math. Mod. Meth. Appl. S. 11 (2001) 521-547. 
[2] P. Angot, C. Bruneau, P. Fabrie, A penalization method to take into account obstacles in incompressible viscous flows., Num. Math. 81 (1999) $497-520$.

[3] J. Haslinger, T. Kozubek, R. Kucera, G. Peichl, Projected Schur complement method for solving non-symmetric systems arising from a smooth fictitious domain approach, Numer. Linear Algebra 14 (2007) 713-739.

[4] G. Evans, A note on the existence of a solution to a problem of Stefan, Q. Appl. Math. 9 (1951) 185-193.

[5] I. Daubechies, Ten lectures on wavelets, SIAM, 1992.

[6] Y. Meyer, Ondelettes et Opérateurs I: Ondelettes, Hermann, 1990.

[7] I. Daubechies, Orthonormal bases of compactly supported wavelets, Appl. Comput. Harmon. Anal. 41 (1988) 909-996.

[8] A. Cohen, Wavelets methods in numerical analysis, handbook of numerical analysis, North-Holland, 2000.

[9] S. Bertoluzza, Interior estimates for the wavelet Galerkin method, Num. Math. 78 (1997) 1-20.

[10] R. Nicolaides, Existence,uniqueness and approximation for generalized saddle point problems, SIAM J. Numer. Anal. 19 (1982) 349-357.

[11] A. Quarteroni, A. Valli, Numerical approximation of partial differential equations, volume 23 of Springer series in computational mathematics, Springer-Verlag, 1997.

[12] J. Baccou, J. Liandrat, Definition and analysis of a wavelet/fictitious domain solver for the 2d-heat equation on a general domain, Math. Mod. Meth. Appl. S. 16 (2006) 819-845.

[13] P. Ponenti, Algorithmes en ondelettes pour la résolution d'equations aux dérivées partielles, Ph.D. thesis, Université de Provence, 1994.

[14] J. Nitsche, A. Schatz, Interior estimates for Ritz-Galerkin methods, Math. Comput. 28 (1974) 937-958. 
[15] S. Lazaar, P. Ponenti, J. Liandrat, P. Tchamitchian, Wavelet algorithms for numerical resolution of partial differential equations, Comp. Meth. App. Mech. Eng. 116 (1994) 309-314.

[16] V. Perrier, C. Basdevant, La décomposition en ondelettes périodiques, un outil pour l'analyse de champs inhomogènes. théorie et algorithmes, La recherche aerospatiale 3 (1989) 57-67.

[17] G. Chiavassa, J. Liandrat, A fully adaptive wavelet algorithm for parabolic partial differential equations, Appl. Numer. Math. 36 (2001) 333-358.

[18] M. Elghaoui, R. Pasquetti, A spectral embedding method applied to the advection-diffusion equation, J. Comput. Phys. 125 (1996) 464-476.

[19] P. Yin, On a wavelet/smooth fictitious domain method for the approximation of the Stefan problem, Ph.D. thesis, Université d'Aix-Marseille, 2011.

[20] A. Cohen, I. Daubechies, P. Vial, Wavelets on the interval and fast wavelet transforms, Appl. Comput. Harmon. Anal. 1 (1993) 54-81.

[21] S. Bertoluzza, S. Falletta, Wavelets on ]0,1[ at large scales, J. Fourier Anal. Appli. 9 (2003) 261-288. 